\title{
Structured clinical examinations in labor: Rekindling the craft of
}

\section{obstetrics}

Johanne Kolvik. Iversen ${ }^{1,2}$, Anne Flem. Jacobsen ${ }^{1,2}$, Thea Falkenberg. Mikkelsen ${ }^{1}$, Torbjørn Moe Eggebø $\varnothing^{3,4}$

${ }^{1}$ Department of Obstetrics and Gynecology, Oslo University Hospital, Norway ${ }^{2}$ Institute of clinical medicine, University of Oslo, Norway ${ }^{3}$ Center for Fetal Medicine, Trondheim University Hospital (St Olavs Hospital), Trondheim, Norway, ${ }^{4}$ Institute of clinical and molecular medicine, Norwegian University of Science and Technology, Trondheim, Norway.

Corresponding author:

Torbjørn Moe Eggebø

Center for Fetal Medicine

Trondheim University Hospital (St Olavs Hospital,

Trondheim, Norway

tme@lyse.net

Telephone number: +4792699433

Running head: Clinical examinations in labor

Key words: Labor, clinical examination, station, position, intrapartum ultrasound

Abbreviations: OA, occiput anterior; OT, occiput transverse; OP, occiput posterior; ROT

(right occiput transverse); LOT, left occiput transverse; BMI, body mass index. 


\section{Abstract}

Objectives: Exact knowledge of fetal station and position is of paramount importance for reliable surveillance of labor progress and a prerequisite for safe operative vaginal procedures. Detailed clinical assessments are thoroughly described in old textbooks, but almost forgotten in contemporary obstetrics. Ultrasound is suggested as an objective diagnostic tool in active labor. Several publications have demonstrated a low correlation between ultrasound and clinical assessment of fetal head station and position, but the methods of clinical assessment in these studies are poorly described. We wanted to explore if a quality clinical assessment could perform better than clinical assessment in previous publications, by analysing the correlation between a structured method of clinical assessment and intrapartum ultrasound.

Methods: In all, 100 laboring women with cervical dilatation $\geq 7 \mathrm{~cm}$ were included in a prospective cohort study at Oslo University Hospital from October to December 2016. The study design was cross sectional. Clinical examinations were performed by one special educated consultant (JKI), and transabdominal and transperineal ultrasound clips were recorded and examined by a blinded expert in intrapartum ultrasound (TME). Fetal position was classified as a clock face with 12 units (hourly divisions) and thereafter categorized as occiput anterior (OA), left occiput transverse (LOT), occiput posterior (OP) and right occiput transverse (ROT) positions. Fetal station was categorized clinically from -5 to +5 and measured with ultrasound as angle of progression (AoP) and head-perineum distance (HPD). AoP is the angle between a longitudinal line through the symphysis and a tangent to the head contour. HPD is the shortest distance between the fetal skull and the perineum.

Results: Eight women were excluded due to strong contractions between clinical assessments and ultrasound measurements, fetal distress or incomplete examinations. Fetal position assessed by ultrasound and clinical examination agreed exactly in 48/92 (52\%) cases, 
within one unit (hour) in 87/92 (95\%) of cases and within two units in 90/92 (98\%) of cases. It differed by three units in one case and by five units in one case. The agreement categorized into OA, LOT, OP and ROT was good (Cohen's kappa 0.72; 95\% CI 0.61-0.84). For station, the agreement was very good for both head-perineum distance (Pearson correlation coefficient $\mathrm{r}=0.86$; $95 \% \mathrm{CI} 0.80-0.91)$ and angle of progression ( $\mathrm{r}=0.77 ; 95 \%$ CI to $0.67-$ 0.84). The correlation between HPD and AoP was good $(\mathrm{r}=0.76 ; 95 \%$ CI $0.65-0.84)$.

Conclusion: We found very good correlations between structured clinical assessments and ultrasound examinations, suggesting that an objective quality in clinical examinations is possible to achieve. More focus on clinical skills training may improve accuracy for clinicians. 


\section{Introduction}

Accurately assessing fetal head position and station are widely accepted prerequisites for assessing progress of labor and safe operative vaginal deliveries. This is traditionally done by manual clinical examinations. However, comparative studies have found low accuracy in simulator studies and low correlation with ultrasound examinations. Error rates of $20 \%$ to $60 \%$ for position [1-5] and more than $30 \%$ for station [6] are published. When compared, senior doctors performed only marginally better than junior doctors [6]. However, older studies found inter-observer agreement for clinical assessment of station to be much higher with almost $90 \%$ agreement [7]. It has been suggested that modern teaching methods for clinical examinations are not adequate [8].

Ultrasound has yielded more consistent results in recent studies, and some argue that it should be the method of choice, foregoing clinical assessment [9]. The clinical assessment yields important additional information, such as degree of flexion and asynclitism, which may be difficult to assess with ultrasound. Ultrasound is not a gold standard, and information can be lost or misinterpreted. A large randomised controlled trial found that ultrasound examinations prior to operative vaginal delivery were more precise than clinical assessments, but no difference in maternal and fetal outcomes were found. This study concluded that an imaging approach alone will not reduce morbidity, and that a more integrated skills-based approach is necessary [10].

Previous studies exploring the correlation between ultrasound and clinical assessment have not provided a thorough description of the method of clinical assessment used. Few clinicians receive any formal training in clinical assessment of the fetal head in labor. We have developed a method of structured manual assessment as part of a larger training program in practical obstetrics. We wanted to explore if this structured clinical assessment performed better than clinical assessment in previous publications, by analysing 
the correlation between this structured method of clinical assessment and intrapartum ultrasound.

\section{Materials and methods}

We conducted a prospective cohort study at Oslo University Hospital from October to December 2016. The hospital has 7300 births yearly, and is a tertiary referral hospital. The study design was cross sectional. Women in active labor and singleton pregnancy, cephalic presentation and $\geq 7 \mathrm{~cm}$ cervical dilatation were included. Exclusion criteria were precipitous labor, fetal distress or obvious labor progress between manual and ultrasound examinations. The regional ethics committee (REK 2016/437) and the local personal data officer (PVO 2016/6668) approved the study. Women were informed about the study at admission to the labor ward and written consent was obtained from all participants at inclusion.

\section{Structured clinical examinations}

A consultant (JKI) with more than ten years' experience in obstetrics performed all clinical examinations in the study. Women were included as consecutive cases when the consultant was on call, and each woman was only examined once. The structured manual assessment started with abdominal examination, using Leopold's manoeuvres to assess lie, position of the fetal back, attitude and level of engagement, given by fifths of the head palpable above the symphysis.

Thereafter, a vaginal examination was performed. Station was assessed using the ischial spine (station 0) as the first reference. The ischial spine was first identified, and the perceived station was then checked against the other anatomical landmarks of the pelvis, namely the ramus tuber ischiadicum (station +3$)$, the sphincter ani muscle $($ station +4$)$ and the symphysis pubis. At station 0 two fingers only barely fit behind the symphysis. Clinically, station was given by the 1988 ACOG definition, with five stations above (-5) and below $(+5)$ the level of the spine ( 0 station) [11]. 
Position was assessed by identifying the sagittal suture, which was followed posteriorly until a fontanel was identified. The fontanel was identified by searching for the frontal suture of the anterior fontanel or lack thereof (posterior fontanel). The sagittal suture was then followed anteriorly, to search for the contralateral fontanel. Both fontanels were always identified, to avoid diagnostic errors and assess flexion. The circumference of the head was then swiped, to search for other landmarks as ears, eyes and nose. Position was classified as a clock face with 12 units (hourly divisions). All manual findings were thoroughly noted before scanning commenced.

\section{Ultrasound examinations}

A transabdominal and a transperineal ultrasound scan were recorded by the same examiner immediately after the clinical examination, but without performing any measurements. The ultrasound examinations were stored as video clips and transferred to a blinded, experienced ultrasound examiner (TME) not involved in the clinical examinations. Three Voluson S8 devices (GE Medical Systems, Zipf, Austria) with 3.5-7.5-MHz 2D curved multifrequency transabdominal transducers were used. Fetal head and spine position were first assessed from the transabdominal recording. Thereafter, angle of progression (AoP), head perineum distance (HPD) and fetal position were assessed by a transperineal scan as described previously [1215]. AoP was measured in the sagittal plane as the angle between the longitudinal axis through the symphysis and the tangent to the head contour [12]. HPD was measured in the axial plane related to the mother (transverse transperineal scanning) as the shortest distance between the outer bony limit of the fetal skull and the perineum $[14,15]$. The transducer was placed in the posterior fourchette and the soft tissue was compressed against the pubic bone until it was not possible to come closer.

Fetal head position was assessed both from the transabdominal and transperineal scanning, and the transperineal approach preferred whenever possible. Position was classified 
in the same way as the clinical examinations as a clock face with 12 units (hourly divisions) and thereafter categorized in accordance with a study by Akmal et al. with positions $\geq 10$ and $\leq 2$ categorized as occiput anterior (OA), 3 as left occiput transverse (LOT), $\geq 4$ and $\leq 8$ as occiput posterior (OP) and 9 as right occiput transverse (ROT) [16]. The same categorization was used in the manual examinations when the two methods were compared.

\section{Statistical analyses}

Categorical variables were compared with chi square test and unweighted Cohen's kappa. Distribution of continuous variables was tested with Kolomogorov-Smirnov test and normality plots. Correlations were tested with linear regression and Pearson correlation coefficient (r). Data were analysed with the statistical software package SPSS statistics version 25.0 (IBM SPSS, Armonk, NY, IMB Corp, USA) and with Vassar Stats (http://vassarstats.net). $\mathrm{P}<0.05$ was considered significant.

\section{Results}

In all, 100 women were included. Three were excluded due to strong contractions with obvious progression between the manual assessment and the ultrasound examination, three were excluded due to fetal distress, and two examinations were incomplete (one due to patient discomfort and one due to a conflicting emergency in the ward). The final study population comprised 92 women. The characteristics of the study population are presented in Table 1.

Fetal position assessed by ultrasound and clinical examination agreed exactly in 48/92 $(52 \%)$ cases, within one unit (hour) in $87 / 92(95 \%)$ of cases and within two units in $90 / 92$ $(98 \%)$ of cases. It differed by three units in one case and by five units in one case. The agreement categorized into OA, LOT, OP and ROT was good (Cohen's kappa 0.72; 95\% CI 0.61-0.84). Details are shown in Table 2.

Fetal stations measured with HPD and AoP were normally distributed. The association between HPD measurements and fetal station assessed with clinical examination is shown in 
Figure 1 . The regression equation was $y=32-4.8 x$ showing that one step change in manually assessed station corresponded to around five mm change in HPD. The correlation was very good $\mathrm{r}=0.86(95 \%$ CI 0.80 to 0.91$)$. Mean HPD for station -3 to +3 was $48 \mathrm{~mm}, 40 \mathrm{~mm}, 37 \mathrm{~mm}$, $32 \mathrm{~mm}, 27 \mathrm{~mm}, 23 \mathrm{~mm}$ and $16 \mathrm{~mm}$, respectively. We had no cases with $-5,-4,+4$ or +5 included.

The association between AoP measurements and fetal station examined clinically is shown in Figure 2 . The regression equation was $y=113+6.5 x$ showing that one step change in manually assessed station corresponded to $6.5 \mathrm{~mm}$ change in AoP. The correlation was good $\mathrm{r}=0.77(95 \% \mathrm{CI}$ to $0.67-0.84)$. Mean AoP for station -3 to +3 was 93 degrees, 97 degrees, 109 degrees, 110 degrees, 119 degrees, 127 degrees and 132 degrees, respectively. The correlation between HPD and AoP was good $(\mathrm{r}=0.76 ; 95 \%$ CI $0.65-0.84)$ and illustrated in Figure 3.

\section{Discussion}

We found a very good correlation between structured clinical examinations and ultrasound measurements. In previous studies, the agreement between clinical assessments and ultrasound measurements was low $[1-6,9,10]$. We found considerably higher agreement. We believe our study, in line with previous publications, demonstrates that structured manual assessment has an objective quality that can be acquired to a high level of accuracy [7]. Evidence supports the fact that simulation training increases other practical skills $[17,18]$, yet to our knowledge, few if any residency programs have made simulation training in manual assessment in labor a compulsory part of their program.

A strength of our study is that the analysis and measurements of the ultrasound examinations was done by an external obstetrician uninvolved in the clinical examination. It may be a limitation that only one consultant performed all clinical examinations, but we intended to compare only one high qualified clinical examiner and one ultrasound expert to 
avoid inter-observer variation. The consultant was probably not representative of her cohort as she currently teaches simulation-based structured manual assessment, but her level of accuracy demonstrates that the structured method of manual assessment has an objective quality that makes it possible to teach. Another limitation is that the clinical consultant also performed the ultrasound acquisitions, but we wanted the interval between clinical examinations and ultrasound recordings to be as short as possible. Clinical findings were noted before the scan, and the consultant did not perform any measurements on the video clips. Some variation in examined head station may be partly due to progression of labor because a contraction sometimes occurred before the scanning was performed.

The international society of ultrasound in obstetrics and gynecology (ISUOG) recommends the use of ultrasound in labor and has published guidelines for ultrasound use [19]. The repeatability in ultrasound measurements is presented in previous studies and found to be good, but still some variations were found [20,21]. We found good correlation between HPD measurements and AoP measurements as also found previously [22]. However, ultrasound has also limitations and cannot be considered a gold standard. Accuracy depends on training and equipment [20]. Classifying position as "four o'clock or five o'clock" is subjective if the position seems to be in the middle, partly explaining variation between ultrasound and manual examinations. Although we strived to keep the interval between examinations as short as possible, contractions sometimes occurred, and some progression in labor may have happened, especially at low stations. The regression equation shows that HPD changes around five $\mathrm{mm}$ for each step in manually assessed station, but the correlation is probably not linear because the birth canal is curved. The corresponding changes in HPD are probably larger at high and low stations than at mid stations due to the shape of the birth canal. 
In our opinion, clinical examination and ultrasound complete each other. Relying solely on ultrasound as some suggest [9] would lower quality, as described in a recent RCT [10]. As the authors concluded: "one potential explanation is that ultrasound enhanced the diagnosis of fetal malpositions, but not the operator's ability to deal with it." This is the crux. A deep understanding of the mechanics of labor is necessary to translate diagnostic findings into practical solutions. If nobody trains new doctors in basic skills, the skills will die out.

Exact knowledge of position and station is of paramount importance before performing an operative vaginal delivery or manual rotation [14, 23-25]. We investigated results for fetal head station and position as these factors are most widely studied, however the clinical examination has other qualities that are difficult to reproduce with ultrasound [8]. To select the best instrument and place it accurately and safely on the fetal skull, the clinician needs to assess not only position and station of the fetal head, but also the asynclitism, degree of flexion, caput succedaneum and moulding. No one should apply an operative instrument on a fetal skull they have yet to touch. A recent systematic review claimed that moulding is of uncertain importance in "contemporary practice" [9], but we argue that knowing the pitfalls when assessing station is of the utmost importance, as described in textbooks and older publications [26-28]. Severe fetal head moulding may cause misdiagnosis when assessing station vaginally or with ultrasound only, as the leading bony part is the reference point. The leading bony part may present below station 0 , whereas the largest diameter of the fetal head is still above the pelvic brim; i.e. in reality high station. In our study, fetal station assessed by clinical examination was based on the combination of abdominal and vaginal clinical findings as thoroughly described in older textbooks $[27,28]$. This will reduce the risk of misdiagnosing high station because of moulding.

If by "contemporary practice" we mean "lack of proficiency", the response should not be to abandon all hope, but to improve our teaching. A tactile discipline requires tactile skills. 
We believe that a structured manual assessment and knowledge of the mechanics of labor, combined with ultrasound in difficult cases, provides a safer frame for both patients and physicians. From this starting point, a rekindling of the art of operative vaginal delivery can begin.

\section{Acknowledgements}

We thank the midwives at Oslo University Hospital Ullevål for their logistical assistance for this study, and David Kenny for help with proofreading the manuscript.

\section{Disclosure statement}

No potential conflict of interest was reported by the authors 


\section{References}

1. Akmal S, Kametas N, Tsoi E, Hargreaves C, Nicolaides KH. Comparison of transvaginal digital examination with intrapartum sonography to determine fetal head position before instrumental delivery. Ultrasound Obstet Gynecol. 2003; 21:437-440.

2. Dupuis O, Ruimark S, Corinne D, Simone T, Andre D, Rene-Charles R. Fetal head position during the second stage of labor: comparison of digital vaginal examination and transabdominal ultrasonographic examination. Eur J Obstet Gynecol Reprod Biol. 2005; 123:193-197.

3. Sherer DM, Miodovnik M, Bradley KS, Langer O. Intrapartum fetal head position I: comparison between transvaginal digital examination and transabdominal ultrasound assessment during the active stage of labor. Ultrasound Obstet Gynecol. 2002; 19:258-263.

4. Sherer DM, Miodovnik M, Bradley KS, Langer O. Intrapartum fetal head position II: comparison between transvaginal digital examination and transabdominal ultrasound assessment during the second stage of labor. Ultrasound Obstet Gynecol. 2002; 19:264-268.

5. Zahalka N, Sadan O, Malinger G, Liberati M, Boaz M, Glezerman M, et al. Comparison of transvaginal sonography with digital examination and transabdominal sonography for the determination of fetal head position in the second stage of labor. Am J Obstet Gynecol. 2005; 193:381-386.

6. Dupuis O, Silveira R, Zentner A, Dittmar A, Gaucherand P, Cucherat M, et al. Birth simulator: reliability of transvaginal assessment of fetal head station as defined by the American College of Obstetricians and Gynecologists classification. Am J Obstet Gynecol. 2005; 192:868-874.

7. Bergsjo $P$, Koss KS. Interindividual variation in vaginal examination findings during labor. Acta Obstet Gynecol Scand. 1982; 61:509-510.

8. Olah KS. Reversal of the decision for caesarean section in the second stage of labour on the basis of consultant vaginal assessment. J Obstet Gynaecol. 2005; 25:115-116.

9. Bellussi F, Ghi T, Youssef A, Salsi G, Giorgetta F, Parma D, et al. The use of intrapartum ultrasound to diagnose malpositions and cephalic malpresentations. Am J Obstet Gynecol. 2017; 217:633-641.

10. Ramphul M, Ooi PV, Burke G, Kennelly MM, Said SA, Montgomery AA, et al. Instrumental delivery and ultrasound : a multicentre randomised controlled trial of ultrasound assessment of the fetal head position versus standard care as an approach to prevent morbidity at instrumental delivery. BJOG. 2014; 121:1029-1038.

11. American College of Obstetricians and Gynecologists. Obstetric forceps. The College, Washington; 1988 (Committee Opinion No: 59).

12. Barbera AF, Pombar X, Perugino G, Lezotte DC, Hobbins JC. A new method to assess fetal head descent in labor with transperineal ultrasound. Ultrasound Obstet Gynecol. 2009; 33:313-319.

13. Ghi T, Farina A, Pedrazzi A, Rizzo N, Pelusi G, Pilu G. Diagnosis of station and rotation of the fetal head in the second stage of labor with intrapartum translabial ultrasound. Ultrasound Obstet Gynecol. 2009; 33:331-336.

14. Kahrs BH, Usman S, Ghi T, Youssef A, Torkildsen EA, Lindtjorn E, et al. Sonographic prediction of outcome of vacuum deliveries: a multicenter, prospective cohort study. Am J Obstet Gynecol. 2017; 217:69 e61-69 e10. 
15. Eggebo TM, Gjessing LK, Heien C, Smedvig E, Okland I, Romundstad P, et al. Prediction of labor and delivery by transperineal ultrasound in pregnancies with prelabor rupture of membranes at term. Ultrasound Obstet Gynecol. 2006; 27:387-391.

16. Akmal S, Tsoi E, Kametas N, Howard R, Nicolaides KH. Intrapartum sonography to determine fetal head position. J Matern Fetal Neonatal Med. 2002; 12:172-177.

17. Kumar A, Sturrock S, Wallace EM, Nestel D, Lucey D, Stoyles S, et al. Evaluation of learning from Practical Obstetric Multi-Professional Training and its impact on patient outcomes in Australia using Kirkpatrick's framework: a mixed methods study. BMJ Open. 2018; 8:e017451.

18. Stone H, Crane J, Johnston K, Craig C. Retention of Vaginal Breech Delivery Skills Taught in Simulation. J Obstet Gynaecol Can. 2018; 40:205-210.

19. Ghi T, Eggebo T, Lees C, Kalache K, Rozenberg P, Youssef A, et al. ISUOG Practice Guidelines: intrapartum ultrasound. Ultrasound Obstet Gynecol. 2018; 52:128-139.

20. Benediktsdottir S, Salvesen KA, Hjartardottir H, Eggebo TM. Reproducibility and acceptability of ultrasound measurements of head-perineum distance. Acta Obstet Gynecol Scand. 2018; 97:97-103.

21. Ghi T, Contro E, Farina A, Nobile M, Pilu G. Three-dimensional ultrasound in monitoring progression of labor: a reproducibility study. Ultrasound Obstet Gynecol. 2010; 36:500-506.

22. Torkildsen EA, Salvesen KA, Eggebo TM. Agreement between two- and threedimensional transperineal ultrasound methods in assessing fetal head descent in the first stage of labor. Ultrasound Obstet Gynecol. 2012; 39:310-315.

23. Graham K, Phipps H, Hyett JA, Ludlow JP, Mackie A, Marren A, et al. Persistent occiput posterior: OUTcomes following digital rotation: a pilot randomised controlled trial. Aust N Z J Obstet Gynaecol. 2014; 54:268-274.

24. Ramphul M, Kennelly MM, Burke G, Murphy DJ. Risk factors and morbidity associated with suboptimal instrument placement at instrumental delivery: observational study nested within the Instrumental Delivery \& Ultrasound randomised controlled trial ISRCTN 72230496. BJOG. 2015; 122:558-563.

25. Kahrs BH, Usman S, Ghi T, Youssef A, Torkildsen EA, Lindtjorn E, et al. Fetal rotation during vacuum extractions for prolonged labor: a prospective cohort study. Acta Obstet Gynecol Scand. 2018;

26. Baxter J. Moulding of the foetal head; a compensatory mechanism. J Obstet Gynaecol Br Emp. 1946; 53:212-218.

27. Dennen EH. Forceps Deliveries, etc. (Second edition.). Philadelphia: F. A. Davis Co., 1964.

28. Kerr JMM. Operative Midwifery. [S.I.]: Bailliere, Tindall and Cox, 1908. 
Table 1 Characteristics of the study population

\begin{tabular}{lcc}
\hline & \multicolumn{2}{c}{$\mathrm{n}=92$} \\
& Median (range) or $\mathrm{n}(\%)$ \\
\hline Maternal characteristics & 32 & $(20-45)$ \\
\hline Maternal age (years) & 61 & $(66)$ \\
Nulliparous women & 22.5 & $(18-35)$ \\
Pre-pregnant BMI & 8 & $(7-10)$ \\
Cervical dilatation (cm) & & \\
Labour characteristics & 25 & $(27)$ \\
\hline Induction of labour & 64 & $(68)$ \\
Epidural analgesia & 63 & $(5.4)$ \\
Oxytocin augmentation & 5 & $(27)$ \\
Cesarean section & 25 & \\
Operative vaginal delivery & & $(2680-4390)$ \\
Characteristics of the newborn & & \\
\hline Birthweight (g) & 3598 & \\
\hline BMI, body mass index & &
\end{tabular}

Table 2 Fetal head position classified with clinical examinations and with ultrasound

\begin{tabular}{llcccc} 
& & \multicolumn{4}{c}{ Clinical examiations } \\
& & OA & LOT & OP & ROT \\
\cline { 2 - 6 } UA & 38 & 3 & 0 & 1 \\
Ultrasound & LOT & 3 & 1 & 2 & 0 \\
examination & OP & 0 & 3 & 37 & 0 \\
& ROT & 2 & 0 & 1 & 1 \\
\cline { 2 - 5 } OA, occiput anterior; LOT, left occiput transverse; \\
OP, occiput posterior; ROT, right occiput transverse
\end{tabular}




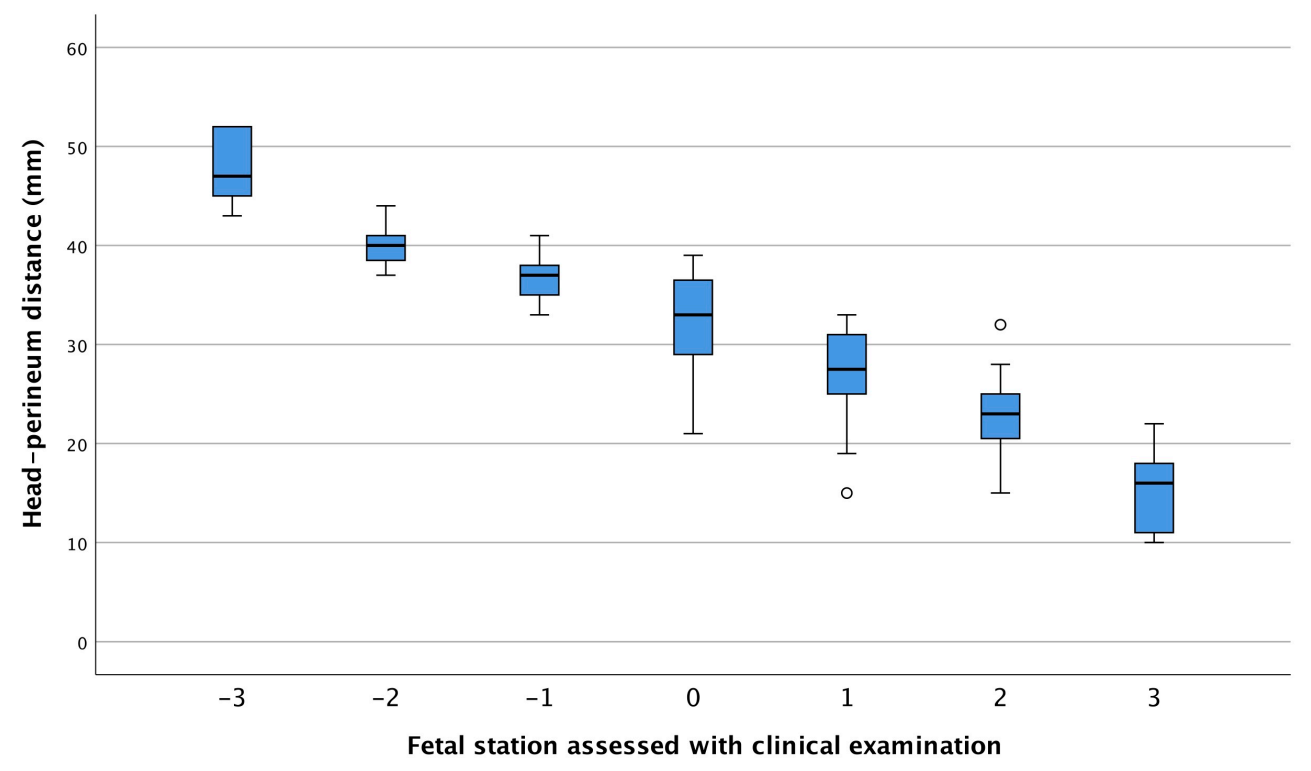

Figure 1. Association between clinical examined station and head-perineum distance.

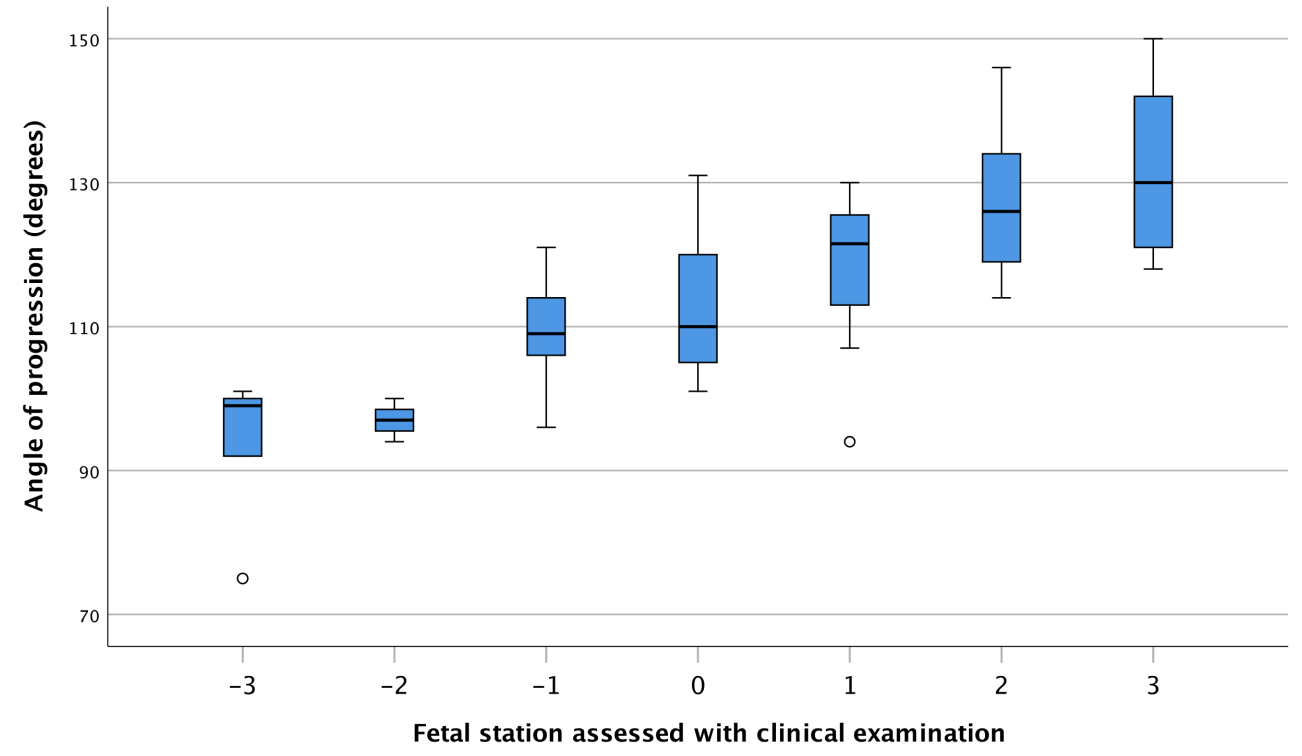

Figure 2. Association between clinical examined station and angle of progression. 


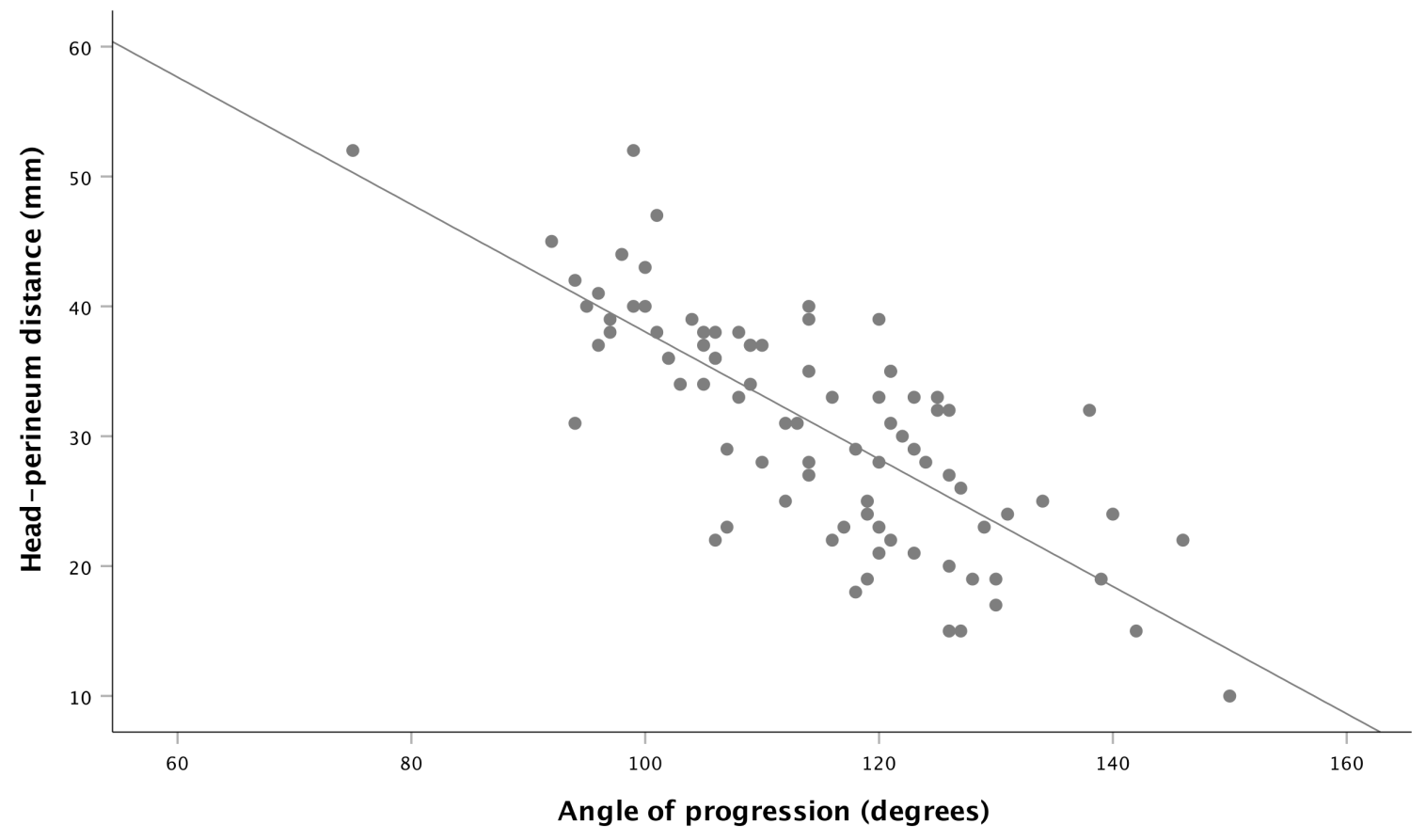

Figure 3. Correlation between head-perineum distance and angle of progression. 